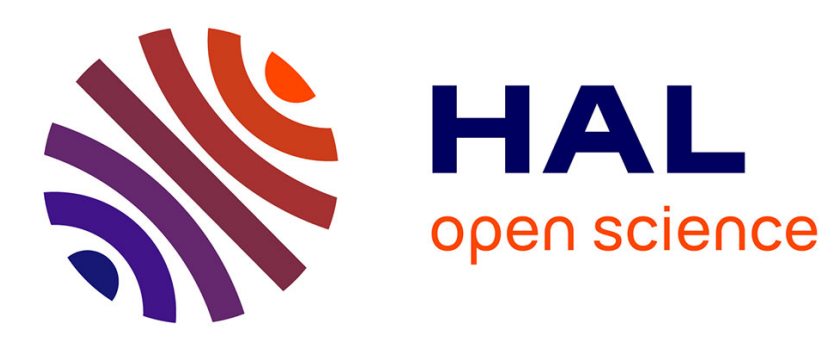

\title{
Etude du comportement d'un système énergétique fonctionnant à partir du couplage des énergies solaire et éolienne
}

\author{
A. Joubert, J. Pécheux
}

\section{- To cite this version:}

A. Joubert, J. Pécheux. Etude du comportement d'un système énergétique fonctionnant à partir du couplage des énergies solaire et éolienne. Revue de Physique Appliquée, 1981, 16 (7), pp.397-403. 10.1051/rphysap:01981001607039700 . jpa-00244932

\section{HAL Id: jpa-00244932 https://hal.science/jpa-00244932}

Submitted on 1 Jan 1981

HAL is a multi-disciplinary open access archive for the deposit and dissemination of scientific research documents, whether they are published or not. The documents may come from teaching and research institutions in France or abroad, or from public or private research centers.
L'archive ouverte pluridisciplinaire HAL, est destinée au dépôt et à la diffusion de documents scientifiques de niveau recherche, publiés ou non, émanant des établissements d'enseignement et de recherche français ou étrangers, des laboratoires publics ou privés. 


\title{
Etude du comportement d'un système énergétique fonctionnant à partir du couplage des énergies solaire et éolienne
}

\author{
A. Joubert et J. Pécheux $(*)$ \\ Laboratoire d'Energétique Solaire, 40, av. du Recteur-Pineau, 86022 Poitiers Cedex, France \\ $\left({ }^{*}\right)$ Laboratoire d'Etudes Aérodynamiques et Thermiques, 40, av. du Recteur-Pineau, 86022 Poitiers Cedex, France
}

(Reçu le 3 février 1981, révisé le 6 avril 1981, accepté le 7 avril 1981)

\begin{abstract}
Résumé. - On modélise globalement une petite centrale utilisant les énergies solaire et éolienne. A partir du bilan énergétique au niveau du captage, des dimensions relatives des modules on cherche une optimisation du stockage ainsi que la probabilité de satisfaction de fournir la demande constituée de besoins de chauffage et d'énergie électrique utilisable localement.
\end{abstract}

\begin{abstract}
A rough modelisation of a small thermal power plant using solar and wind energy is presented. We determine an optimized storage capacity, and the probability to satisfy the desired local demand as a function of the meteorological conditions and the dimensions of the collector modules.
\end{abstract}

1. Introduction. - L'énergie récupérable par une installation solaire est la moyenne temporelle des valeurs instantanées du rayonnement capté, elle est faible et intermittente à cause des périodes non ensoleillées et nocturnes.

L'association de l'énergie éolienne à l'énergie solaire permet de rendre a priori moins aléatoire les variables d'entrée d'une centrale vent-soleil, dans la mesure où ces diverses formes d'énergie ne sont pas corrélées.

L'intérêt d'un tel couplage est, bien sûr, d'avoir plus d'énergie, mais, comme pour tout système solaire, le risque demeure d'avoir trop d'énergie à certains moments et pas assez à d'autres (absence de vent par ciel couvert par exemple). Il s'agit donc, par l'intermédiaire d'une masse thermique tampon-stockage, d'emmagasiner l'excès énergétique lorsqu'il existe et de le restituer lors des périodes de manque d'apport.

A partir des données météorologiques réelles, on simule le fonctionnement d'une mini-centrale pour un ensemble de contraintes fixé. On détermine l'optimum du stockage qui correspond à la meilleure corrélation entre vent, soleil et stock, les problèmes du dimensionnement et de gestion dépendant du site considéré. Le but de cette étude est d'apprécier le complément énergétique dû au vent de l'installation de type vent-soleil.

2. Fonctionnement du système. - 2.1 PrésentaTION ET HYPOTHÈSES. - La centrale étudiée comporte deux modules de captage. Le premier, purement solaire, est constitué de miroirs concentrateurs réflé- chissant le rayonnement direct reçu sur une chaudière. Le second module se compose de plusieurs éoliennes couplées entre elles de façon à fournir une énergie relativement constante dans toute la gamme des vitesses du vent.

Ces modules sont reliés au stockage et à la demande comme le schématise la figure 1 .

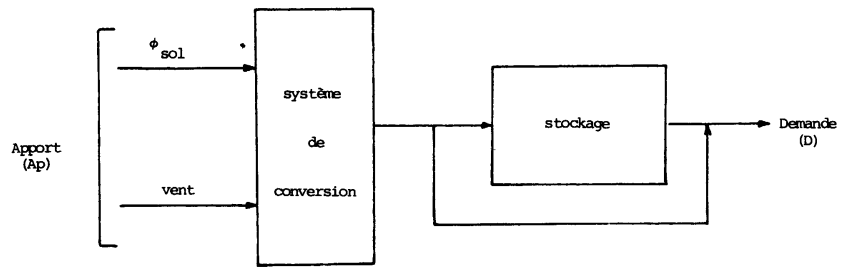

Fig. 1. - Modèle schématique du système étudié.

[Schematic model of studied system.]

Dans le cas où l'apport $A_{\mathrm{p}}$ et la demande $D$ sont du même ordre de grandeur, le stockage n'est pas utilisé. Lorsque $A_{\mathrm{p}}$ est supérieur à $D$ il y a en même temps recharge du stock et, pour $A_{\mathrm{p}}$ inférieur à $D$ il y a déstockage.

La demande consiste à fournir les besoins en chauffage d'un nombre variable de locaux, identiques à un habitat de référence caractérisé par son volume $V_{0}$ et un coefficient de déperdition volumique global $G_{0}$, ainsi qu'une certaine puissance électrique fixée, directement utilisable, par exemple, par une entreprise locale. Les besoins en chauffage dépendent de la température 
extérieure $T_{\text {ext }}$ et la demande est donc fonction de cette variable météorologique.

L'étude consiste à effectuer des simulations numériques à partir des données météorologiques sur un modèle mathématique du système. La modélisation est relativement globale car la durée des simulations est grande (elle correspond aux sept mois de la période de chauffage légale : $1^{\text {er }}$ octobre- 30 avril). Le pas de temps est égal à la journée et on détermine le taux de couverture du système sur toute la période d'étude.

Une journée solaire moyenne peut, par l'intermédiaire de la centrale, fournir de l'énergie si la durée d'insolation est continue. Par contre, il se peut qu'on ne récupère rien si les périodes d'ensoleillement sont inférieures ou égales au temps d'inertie du système (en particulier de la chaudière). On suppose donc, qu'avec un pas de temps égal à la journée, les moyennes des variables météorologiques sont représentatives de cette journée et que le rayonnement direct est également réparti sur la durée d'insolation. De telles hypothèses justifient la schématisation du système par les formules globales utilisées par la suite.

Le type de stockage n'est pas explicitement défini (forme, volume, nature du fluide); le raisonnement se fait à partir du transfert des quantités d'énergie, les pertes étant proportionnelles à la capacité énergétique instantanée de ce stock. Elles sont approximées par la relation :

$$
P_{\mathrm{t}}=A\left(E_{\mathrm{m}}\right)^{2 / 3}
$$

où $P_{\mathrm{t}}$ représente les pertes thermiques au réservoir,

$E_{\mathrm{m}}$ étant l'énergie maximale du réservoir de stockage à l'instant considéré.

Le coefficient $A$ est déterminé par comparaison avec les valeurs expérimentales des pertes dans le stockage de la petite centrale d'Odeillo. On envisage plusieurs capacités de stockage ( 0 à 12 jours) de manière à déterminer une durée optimale de celui-ci compte tenu des contraintes imposées. La capacité de stockage, exprimée en nombre de jours, correspond à la quantité d'énergie moyenne journalière fournie par le rayonnement direct, multipliée par le nombre de jours considéré.

La répartition des charges se fait comme suit :

a) d'abord satisfaire dans la mesure du possible les besoins en chauffage

$b)$ et, une fois les besoins précédents assurés, fournir éventuellement la demande en électricité. On calcule ensuite la probabilité de satisfaire $(b)$ lorsque la condition (a) est réalisée.

2.2 ENERgIE D'ORIGINE SOlAire. - Le module solaire de l'installation utilise des miroirs concentrateurs au niveau du captage (type centrale d'Odeillo) ne réfléchissant que le rayonnement direct. Dans la plupart des cas [1], on ne dispose que des valeurs du rayonnement global et non du rayonnement direct. Il est donc nécessaire de l'estimer à partir des données de la durée d'insolation journalière et du rayonnement global [2]. (On ne tient pas compte de la nébulosité.) L'apport énergétique solaire $E_{\mathrm{s}}$ est défini par :

$$
E_{\mathrm{s}}=\varphi_{\mathrm{sol}} \cdot S \cdot \eta
$$

où $\varphi_{\text {sol }}$ est le flux direct normal à la surface de captage $S$, $\eta$ étant le rendement total du module solaire de la centrale.

2.3 ENERGIE ÉOLIENNE $E_{v}$ - - Elle est proportionnelle au nombre d'éoliennes $N$ considéré, à l'aire balayée par l'hélice de l'une d'elle $A$ et au cube de la vitesse du vent $V$ [3], [4] :

$$
E_{\mathrm{v}}=\frac{1}{2} \rho C_{\mathrm{p}} A V^{3} \eta_{\mathrm{c}} N=k_{2}^{\prime} V^{3}
$$

où $\rho$ représente la masse volumique de l'air, $C_{\mathrm{p}}$ le coefficient de puissance et $\eta_{\mathrm{c}}$ le rendement du convertisseur couplé à l'éolienne.

Le coefficient de puissance aussi appelé facteur de conversion est le rapport de la puissance fournie par l'éolienne à la puissance théorique que peut fournir l'écoulement libre de l'air à travers la surface $A$. Les performances d'une éolienne donnée (forme, rayon des pales, nombre de pales, ...) dépendent de la vitesse du vent comme le montre la figure $2 a$.

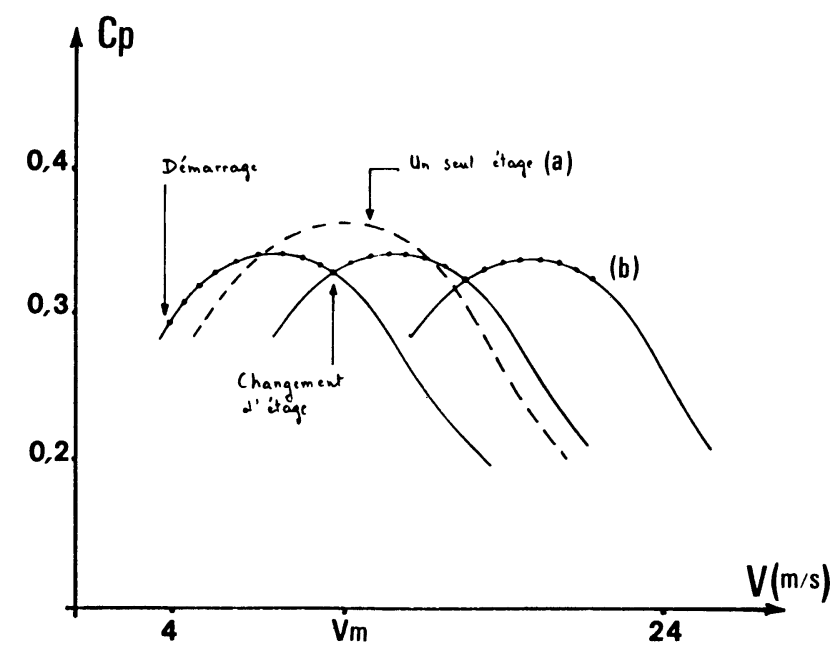

Fig. 2. - Variation du coefficient de puissance en fonction de $V$ d'une seule éolienne $(a)$ et de trois étages couplés $(b)$.

[Variation of the conversion factor versus $V$ for $(a)$ one wind mill (b) for three coupled stages.]

Le facteur de conversion présente un maximum $C_{\mathrm{p} \max }$ qui correspond au meilleur rendement de l'éolienne pour une vitesse du vent égale à $V_{\mathrm{m}}$. Si l'on considère plusieurs éoliennes montées en étages, c'est-à-dire dont leurs caractéristiques présentent des maxima pour différentes valeurs de la vitesse du vent, on obtient la configuration de la courbe $(b)$ de la figure 2. Dans ce cas, le rendement est légèrement diminué mais la centrale éolienne présente un coefficient de puissance pratiquement constant quelle que 
soit la vitesse du vent. C'est l'intérêt primordial du couplage d'éoliennes définies en fonction de la courbe des fréquences du vent du lieu choisi. Dans cette étude les inerties de démarrage des éoliennes ne sont pas prises en compte.

2.4 Puissanç: De Chauffage. - On estime les besoins en chauffage $P_{\text {chauff }}$ d'un habitat par les pertes thermiques de celui-ci et on utilise la formule approximative suivante [5] :

$$
P_{\text {chauff }}=G V\left(T_{\text {nc }}-T_{\text {ext }}\right)=K_{1}^{\prime}\left(T_{\text {nc }}-T_{\text {ext }}\right)
$$

avec $G$ : coefficient de déperdition volumique global,

$V$ : volume des locaux,

$T_{\text {ext }}:$ température extérieure,

$T_{\text {nc }}$ : température de non-chauffage souvent prise entre 15 et $18^{\circ} \mathrm{C}$ car on considère que les apports internes dus aux occupants, aux appareils électriques et aux apports solaires passifs maintiennent la température intérieure des locaux aux environs de $19{ }^{\circ} \mathrm{C}$.

2.5 Stockage ET ÉNERGIE DisPonible. - Des équations (2), (3) et (4) précédentes, on déduit la puissance disponible pour l'industrie $P_{\mathrm{i}}^{\prime}$ et le stockage (5). $\mathrm{Si}$ $E_{\text {stock }}$ est l'énergie du stockage, alors $\mathrm{d} E_{\text {stock }} / \mathrm{d} t$ représente l'énergie stockée (si $\mathrm{d} E_{\text {stock }} / \mathrm{d} t>0$ ) ou déstockée $\left(\mathrm{d} E_{\text {stock }} / \mathrm{d} t<0\right)$

$\varphi_{\text {sol }} S \eta+k_{2}^{\prime} V^{3}=k_{1}^{\prime}\left(T_{\mathrm{nc}}-T_{\mathrm{ext}}\right)+\frac{\mathrm{d} E_{\text {stock }}}{\mathrm{d} t}+P_{\mathrm{i}}^{\prime}$.

Soit, par unité de surface de captage et de rendement solaires :

$P_{\mathrm{i}}=\varphi_{\mathrm{sol}}+k_{2} V^{3}+k_{1}\left(T_{\mathrm{ext}}-T_{\mathrm{nc}}\right)+\frac{\mathrm{d} E}{\mathrm{~d} t}(\gtrless 0)$.

Remarque : Les coefficients $k_{1}$ et $k_{2}$, inversement proportionnels au rendement et à la surface de captage solaires de l'installation, sont respectivement fonction du coefficient de déperdition, du volume des locaux à chauffer, et de la puissance éolienne installée.

Les coefficients de conversion entre les différentes énergies sont compris, pour la transformation énergie mécanique-énergie thermique et électrique, dans l'expression du rendement du convertisseur $\eta_{\mathrm{c}}$ couplé à l'éolienne, et pour la transformation énergie thermique-énergie électrique, dans le rendement du module solaire $\eta$. Les variations de ces coefficients se traduisent par des modifications des valeurs des coefficients globaux $k_{1}$ et $k_{2}$.

3. Résultats. - Ils ont été obtenus en faisant tourner le programme sur sept ou neuf années puis moyennés sauf pour la station de Carpentras où les données étaient limitées.

3.1 ANALYSE DÉTAILléE : EXEMPLE SUR UNE ANNÉE CARACTÉRISTIQUE DU CLIMAT DE LA ROCHELlE. Les courbes de la figure 3 représentent la probabilité
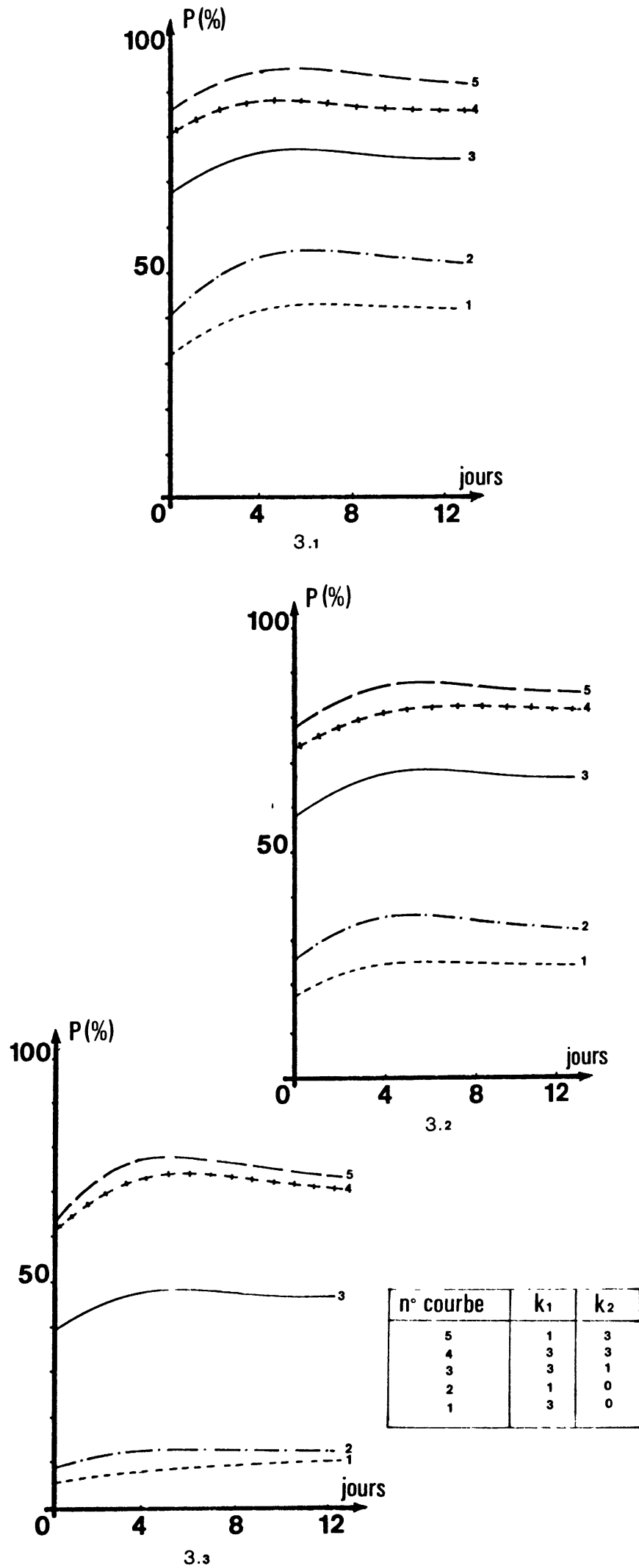

Fig. 3. - Probabilité de satisfaction de la demande locale en fonction de la durée du stockage à La Rochelle pour différentes valeurs de la puissance électrique demandée $P_{\mathrm{i}}$. $3.1 P_{\mathrm{i}}=50 \mathrm{~W}$. $3.2 P_{\mathrm{i}}=75 \mathrm{~W}$. $3.3 P_{\mathrm{i}}=125 \mathrm{~W}$.

[Probability of satisfying the local demand as a function of the storage period in La Rochelle for different values of needed electrical power $P_{\mathrm{i}}, 3.1 P_{\mathrm{i}}=50 \mathrm{~W} .3 .2 P_{\mathrm{i}}=75 \mathrm{~W} .3 .3 P_{\mathrm{i}}=125 \mathrm{~W}$.] 
de satisfaire la demande en électricité, les besoins en chauffage étant assurés, sur la période de chauffe en fonction du nombre de jours de stockage. On remarque que la durée optimale du stock est d'environ quatre jours. Au-delà, les pertes thermiques sont importantes et les probabilités diminuent. Pour une capacité énergétique du stockage faible, les pertes sont pratiquement nulles et la courbe des probabilités croît de façon monotone.

Plus l'écart entre deux courbes de même coefficient de chauffage $k_{1}$ est grand, plus l'apport énergétique éolien est important.

De même, pour une valeur fixée de $k_{2}$, on observe une diminution moyenne de la probabilité de satisfaction de $7 \%$ lorsque $k_{1}$ varie de 1 à 3 , c'est-à-dire lorsqu'on triple le nombre de locaux à chauffer.

Pour un accroissement relatif $\Delta P_{\mathrm{i}} / P_{\mathrm{i}}$ de la puissance nécessaire à l'industrie égal à $50 \%$, la probabilité de satisfaction du système diminue de 10 à $20 \%$ (Fig. 3.1 et 3.2 pour les mêmes coefficients $k_{1}$ et $k_{2}$ ). Pour les valeurs de $k_{1}$ et $k_{2}$ de 3 et 1 respectivement, et pour une puissance électrique demandée de $50 \mathrm{~W}$, le rapport entre la production moyenne et la demande moyenne dépasse $75 \%$ pour une capacité de stockage de 4 jours (Fig. 3.1) à La Rochelle. Elle n'est plus que de $55 \%$ pour une puissance de $125 \mathrm{~W}$ (Fig. 3.3).

$E_{+}$représentant l'énergie électrique moyenne fournie $\left(\right.$ en $\left.\mathrm{kWh} / \mathrm{m}^{2}\right)$ par le système et $E_{-}$l'énergie

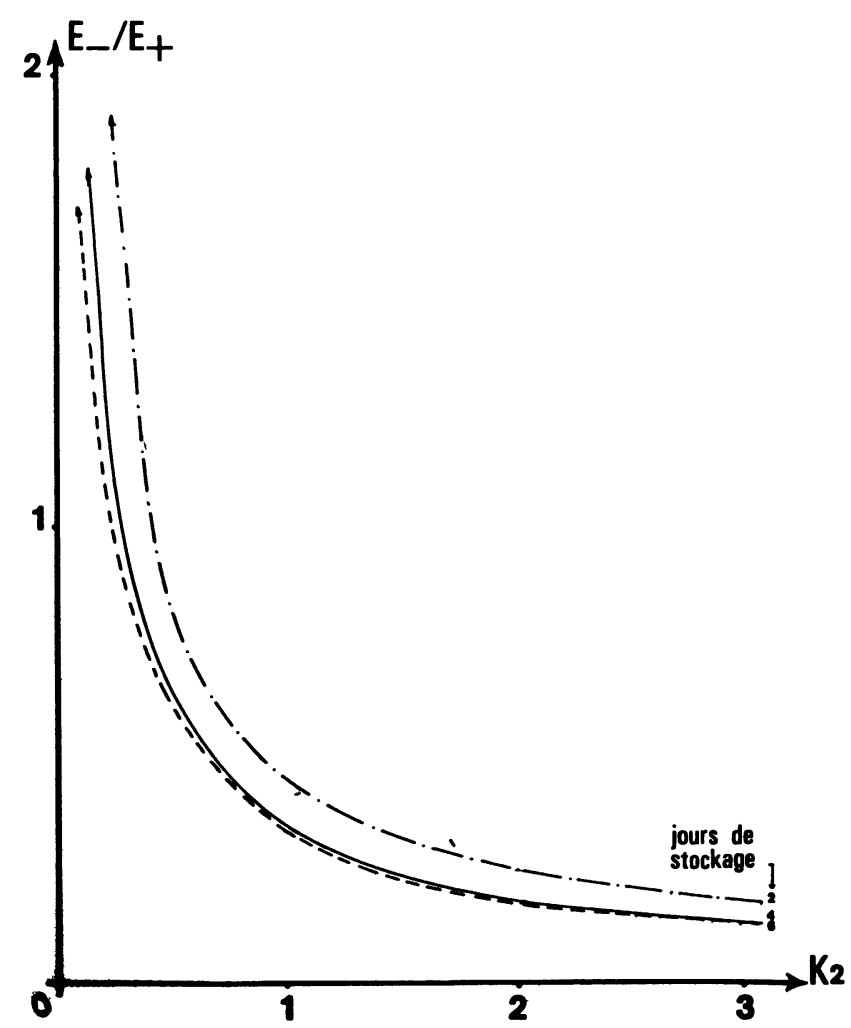

Fig. 4. - Evolution du rapport énergétique $E_{-} / E_{+}$en fonction du coefficient éolien $k_{2}$ pour différentes valeurs de la durée du stockage et pour $k_{1}=1,4$.

[Evolution of energetic ratio $E_{-} / E_{+}$as a function of the wind module coefficient $k_{2}$ for different values of storage period for $k_{1}=1.4$.] d'appoint nécessaire pour satisfaire la demande, on en déduit le rapport $E_{-} / E_{+}$en fonction du coefficient éolien $k_{2}$. La courbe de la figure 4 montre l'évolution de ce rapport pour diverses valeurs du nombre de jours de stockage et pour une valeur de $k_{1}$ égale à 1,4. On remarque la diminution de l'écart entre les courbes pour des durées de stockage voisines de quatre jours.

Les phénomènes observés à La Rochelle sont particuliers dans la mesure où cette station est en bordure de mer et possède de ce fait un micro-climat spécifique (brise de mer et de terre). Il en serait de même pour toute station située dans une bande côtière de quelques dizaines de kilomètres.

\subsection{COMPARAison ENTRE Différentes STATIONS} FRANÇAISES. - 3.2.1 La Rochelle et Carpentras. Du fait des données météorologiques restreintes de Carpentras, nous n'avons pu faire la comparaison avec La Rochelle que sur une période de 100 jours. Bien que, pour cette station, le rayonnement direct y soit mesuré, d'où une meilleure précision que s'il avait dû être estimé, les résultats peuvent ne pas être très probants.

La figure 5 représente la probabilité de satisfaction en fonction de la durée du stockage pour les deux stations considérées. On note un meilleur rendement à Carpentras qu'à La Rochelle, les probabilités pour les mêmes valeurs des coefficients y étant plus élevées (différence entre les courbes de même numéro des figures 5.1 et 5.2 ).

L'étalement des courbes de La Rochelle de la figure 3.1 est plus important que celui de Carpentras (Fig. 5.2) c'est-à-dire que l'apport éolien y est plus net relativement à l'apport solaire.

Le pourcentage d'énergie électrique apporté par la centrale vent-soleil varie entre 76 et $95 \%$ pour La Rochelle et entre 94 et $97 \%$ pour Carpentras comme le montre le tableau I.

Les courbes de la figure 6 montrent l'évolution du rapport des énergies $E_{-} / E_{+}$en fonction des dimensions relatives du module éolien $k_{2}$. La pente des courbes est proportionnelle à l'apport dû au vent qui est supérieur pour La Rochelle. Les valeurs du rapport $E_{-} / E_{+}$pour $k_{2}$ nul sont celles d'une petite centrale de type solaire pur.

Tableau I. - Pourcentages d'énergie électrique fournie par le système à La Rochelle et Carpentras pour une durée de stockage de quatre jours.

[The percentage of electrical energy produced by the system over a four days time-period in La Rochelle and Carpentras.]

$\begin{array}{ccc}k_{2} & \text { La Rochelle } & \text { Carpentras } \\ \overline{-} & - & - \\ 1 & 0,41 & 0,81 \\ 3 & 0,76 & 0,94 \\ & 0,95 & 0,97\end{array}$



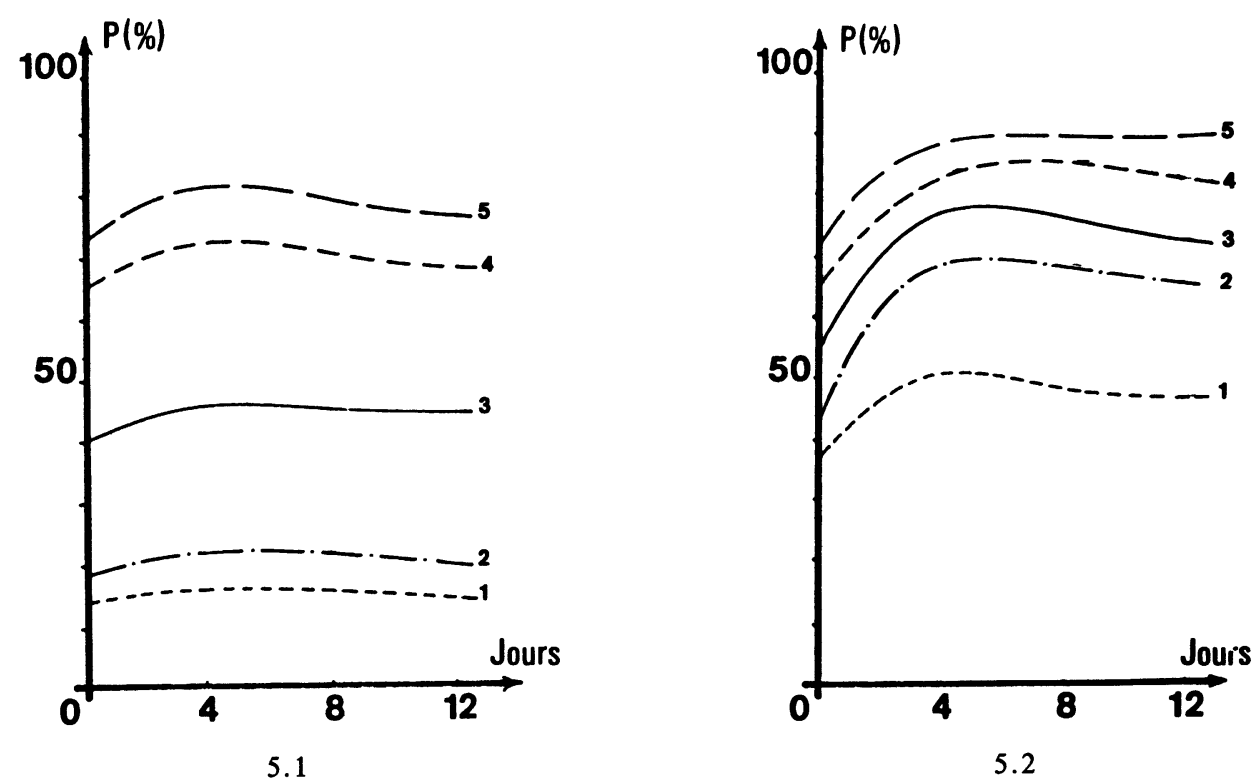

Fig. 5. - Probabilité de satisfaction en fonction de la durée de stockage pour : 1. La Rochelle; 2. Carpentras.

[Probability of satisfying the demand as a function of the storage period for : 1. La Rochelle; 2. Carpentras.]

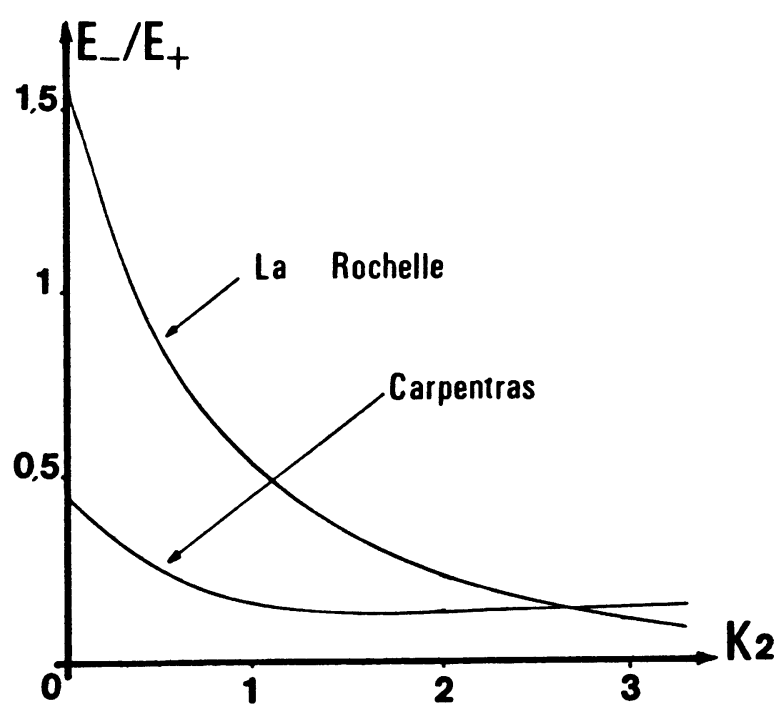

Fig. 6. - Comparaison du rapport $E_{-} / E_{+}$en fonction de $k_{2}$ entre La Rochelle et Carpentras.

[Comparison of $E_{-} / E_{+}$versus $k_{2}$ between La Rochelle and Carpentras.]

En conclusion, on a des rendements supérieurs pour Carpentras, situé dans une région privilégiée du point de vue ensoleillement, mais une médiocre complémentarité vent-soleil. Il y a donc corrélation entre ces deux variables météorologiques.

A La Rochelle, l'apport éolien est important vis-à-vis de l'apport solaire et la corrélation entre vent et soleil apparait meilleure essentiellement pour la raison que l'apport solaire y est relativement faible. Une installation type vent-soleil possédant une valeur de $k_{2}$ égale à 1,25 située à La Rochelle aurait le même rendement qu'une centrale solaire à Carpentras.
3.2.2 Stations de St-Quentin, Rennes, Nancy et Millau comparés à La Rochelle. - Cette étude a été faite à besoins d'énergie de chauffage constants, c'est-à-dire que, si on chauffe dix maisons (de volume $V_{0}=300 \mathrm{~m}^{3}$ ) à Nancy on chauffera respectivement environ dix, onze, douze et quatorze maisons identiques à Millau, St-Quentin, Rennes et La Rochelle. Avec La Rochelle comme référence, chauffer dix maisons revient à chauffer sept maisons à Millau, Nancy et St-Quentin et neuf à Rennes.

Le rapport entre l'énergie fournie et l'énergie nécessaire pour satisfaire la demande est, sur la période de chauffage :

$$
\frac{E_{+}}{D}=\left(1+\frac{E_{-}}{E_{+}}\right)^{-1}
$$

Les variations de ce rapport en fonction du coefficient éolien sont représentées sur la figure 7. On remarque une analogie de comportement pour les trois stations de La Rochelle, Rennes et St-Quentin alors que Nancy et Millau ont des comportements différents. Ceci est en relation avec le climat de ces sites où La Rochelle, Rennes et St-Quentin bénéficient d'un climat océanique alors que Nancy et Millau ont respectivement un climat subcontinental et de montagne.

A Nancy on note un fort apport éolien et des rendements assez faibles puisque avoisinant $50 \%$ pour un coefficient éolien de 1 . L'allure de la courbe de Millau se rapproche de celle obtenue pour Carpentras.

Sans installation éolienne, c'est-à-dire pour $k_{2}$ nul, les rendements se classent proportionnellement à la durée d'insolation hivernale moyenne des stations 


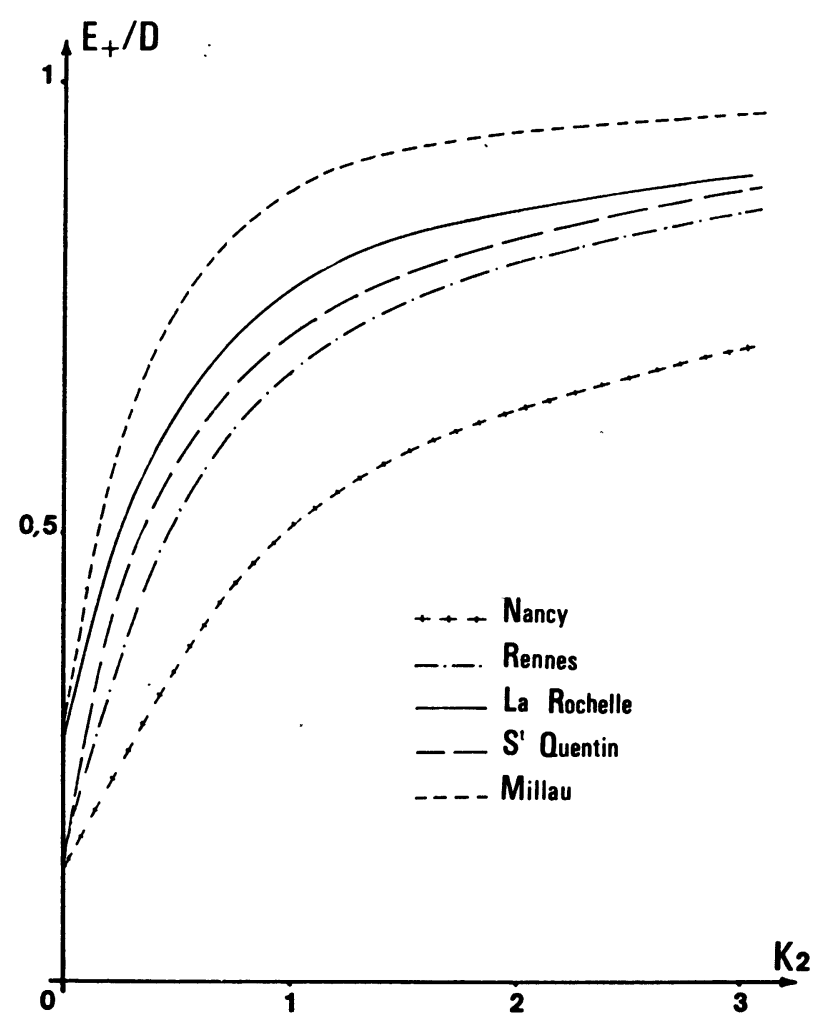

Fig. 7. - Variations du rapport $E_{+} / D$ en fonction de $k_{2}$.

[Variation of ratio $E_{+} / D$ versus $k_{2}$ ]

considérées. Ce résultat, tributaire des hypothèses faites au niveau de la modélisation, est logique du fait que le module solaire n'utilise que le rayonnement direct lui-même proportionnel à la durée d'insolation.

Les pourcentages d'énergie électrique apportée par le système sont groupés dans le tableau II pour la durée optimale du stockage et pour différentes valeurs de $k_{2}$. Ils évoluent de 57 à $97 \%$ en fonction des valeurs croissantes du module éolien $k_{2}$. Pour une valeur de $k_{2}$ égale à 0 on a l'apport solaire relatif à la demande $E_{+\mathrm{s}} / D$ puisque l'énergie totale fournie $E_{+}$ est la somme des énergies fournies par les modules solaires $\left(E_{+\mathrm{s}}\right)$ et éolien $\left(E_{+\mathrm{v}}\right)$. Pour un $k_{2}$ différent de 0 on observe l'apport total de l'installation et on en déduit le rapport entre l'énergie d'origine éolienne et l'énergie d'origine solaire $E_{+\mathrm{v}} / E_{+\mathrm{s}}$ (Fig. 8). Il peut atteindre des valeurs notables, par exemple pour

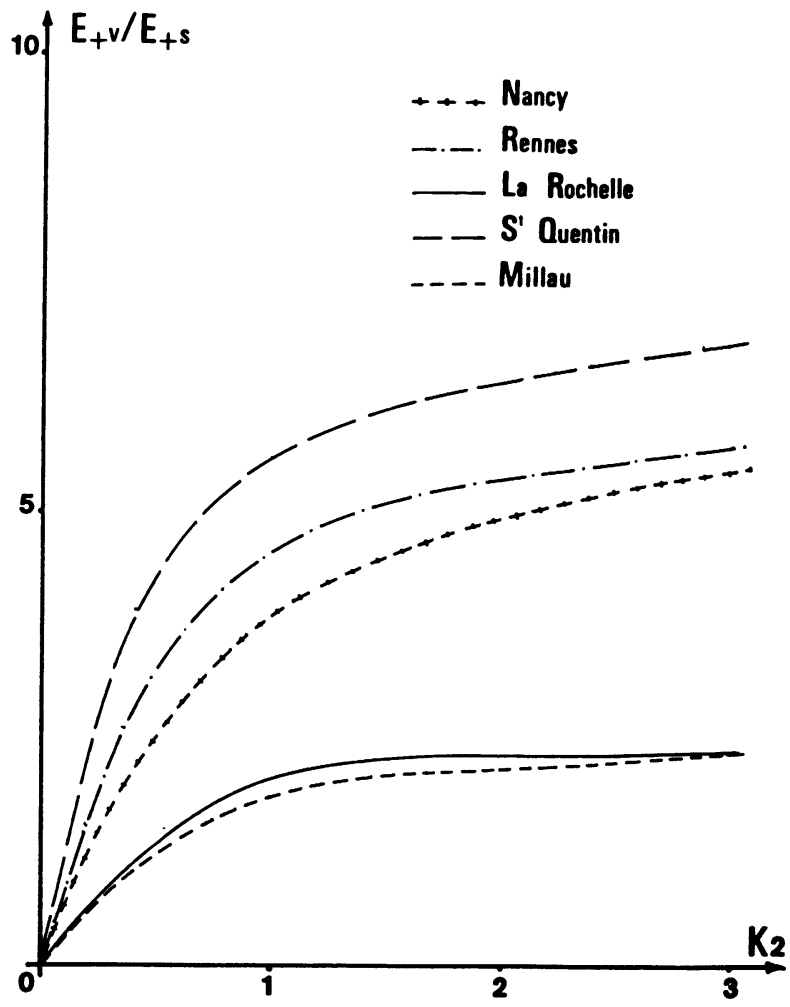

Fig. 8. - Evolution du rapport énergétique $E_{+\mathrm{v}} / E_{+\mathrm{s}}$ en fonction du coefficient éolien $\boldsymbol{k}_{\mathbf{2}}$.

[Evolution of energetic ratio $E_{+\mathrm{v}} / E_{+\mathrm{s}}$ as a function of wind module coefficient $k_{2}$.]

St-Quentin où il est supérieur à 6. Les stations de La Rochelle et Millau ont un comportement analogue et, pour un dimensionnement du module éolien $k_{2}$ égal à 1 , l'énergie éolienne apporte les deux tiers de la fourniture énergétique. Par apport éolien croissant, les stations sont ensuite respectivement Nancy, Rennes et St-Quentin.

Pour des valeurs du coefficient $k_{2}$ élevées, l'apport énergétique d'origine éolien est important vis-à-vis de l'apport solaire et satisfait la presque totalité des besoins. Le rapport $E_{+\mathrm{v}} / E_{+\mathrm{s}}$ tend vers une valeur limite correspondant, pour une station, à la fourniture totale par la seule énergie éolienne de la demande diminuée de l'apport solaire; ce qui explique la

Tableau II. - Pourcentages d'énergie électrique apportée par la minicentrale des stations considérées pour un stockage de quatre jours et à énergie de chauffage constante.

[Percentage of electrical energy supplied by the considered small plants for a storage period of four days and constant heating energy.]

$\begin{array}{cccccc}k_{2} & \text { La Rochelle } & \text { Millau } & \text { Nancy } & \text { Rennes } & \text { St-Quentin } \\ \overline{0} & - & - & - & - & - \\ 1 & 0,35 & 0,37 & 0,16 & 0,21 & 0,18 \\ 2 & 0,83 & 0,92 & 0,57 & 0,76 & 0,78 \\ 3 & 0,90 & 0,96 & 0,69 & 0,86 & 0,88 \\ & 0,93 & 0,97 & 0,76 & 0,89 & 0,91\end{array}$


tendance des courbes à marquer un palier pour les grandes valeurs de $k_{2}$.

4. Conclusion. - Les résultats ont été obtenus à partir de simulations numériques effectuées avec des données météorologiques enregistrées sur plusieurs années. Il ressort que la durée optimale du stockage; quelle que soit la station considérée, est approximativement de l'ordre de quatre jours, où une journée de stockage est définie comme étant la quantité moyenne de l'énergie solaire directe reçue par une surface unité sur la période considérée. Il apparait que le stockage à plus long terme, dans l'état actuel des techniques de l'isolation, n'arrive pas à compenser les pertes dues à de telles durées.

La modélisation globale utilisée pour obtenir ces résultats constitue une première approche de l'étude des phénomènes excluant une précision fine du comportement du système. Elle permet, à l'aide d'un programme simple d'utilisation et de temps de calcul relativement court, de déterminer grossièrement les apports des diverses formes d'énergie. La part de l'énergie d'origine éolienne est deux fois supérieure à l'énergie solaire pour les stations de La Rochelle et Millau alors qu'elle y est respectivement cinq et six fois supérieure pour les autres stations. Dans une seconde approche plus précise, il conviendrait d'utiliser, surtout pour le vent, des relevés effectués à l'endroit exact de l'implantation éventuelle de la centrale.

Cette première approche du couplage des énergies solaire et éolienne montre que cette dernière, encore relativement inexploitée, peut s'avérer de grande utilité dans une petite installation vent-soleil.

\section{Bibliographie}

[1] Fichiers météorologiques de la Météorologie Nationale.

[2] Perrin De Brichambaut, C., Supplément aux cahiers A.F.E.D.E.S. $\mathrm{n}^{\circ} 1$.

[3] Justus, C. G., Vent et performances des éoliennes, traduit et adapté par J. L. Plazy (1980) collection Energie Solaire.

[4] Franklin Institute Press, Energie éolienne. Traduction de J. De- bry, Préface de J. Bonnin (1980), collection Energie Solaire.

[5] Contribution à l'étude des systèmes énergétiques solaires. Application au chauffage de l'habitat, Thèse de $3^{\mathrm{e}}$ cycle de E. Plancq (1979). 